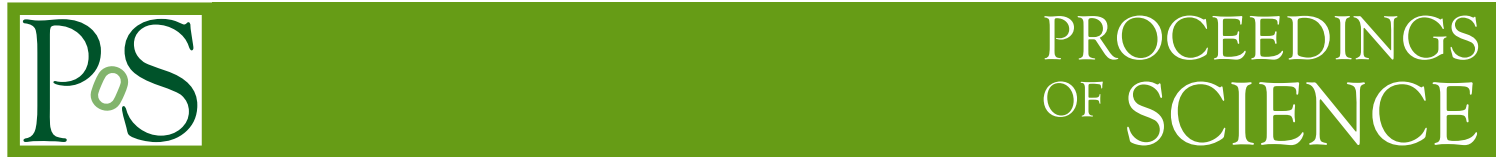

\title{
Experimental Overview on GPDs and TMDs
}

\section{Haiyan Gao*}

Duke University and Duke Kunshan University

E-mail: gaodphy.duke.edu

\section{Tianbo Liu}

Duke University and Duke Kunshan University

E-mail: Eianbe. liudaduke.edu

The generalized parton distribution (GPD) functions and the transverse momentum dependent parton distribution (TMD) functions provide three-dimensional tomographies in the combined momentum-coordinate space (GPD) and the complete momentum space (TMD) of partons inside the nucleon. They uncover the rich dynamics of $\mathrm{QCD}$, and provide qualitative and quantitative information about the partonic orbital angular momentum, an important piece to the solution of the proton spin puzzle. In the last ten years or so, significant progresses have been made experimentally in accessing the GPDs and TMDs. In this talk, we review the experimental progress on GPDs and TMDs and also discuss upcoming experiments in the coming decade.

The 26th International Nuclear Physics Conference

11-16 September, 2016

Adelaide, Australia

\footnotetext{
${ }^{*}$ Speaker.
} 


\section{Introduction}

Nucleons are known as the fundamental building blocks of the visible matter. The point-like constituent particles inside the proton discovered in the 1960s from the deep inelastic scattering experiment at SLAC led to the establishment of the quark-parton model and the experimental foundation for the development of Quantum Chromodynamics (QCD). QCD, a gauge theory with quarks and gluons as the underlying degrees of freedom, is the accepted theory of strong interaction with asymptotic freedom and confinement as two important features. While QCD has been extremely well tested at high energy scales by experiments where perturbative calculations can be carried out and be compared with, an analytical solution to the QCD Lagrangian in the nonperturbative region is notoriously difficult and out of reach. As such our knowledge about how QCD works in the confinement region, where the strong coupling constant is strong, is rather poor. Nucleons, building blocks of atomic nuclei, provide a natural and an effective laboratory for physicists to study QCD in the confinement region.

The structure of nucleons is a rich and vibrant research area, which involves studies of the ground state properties (mass, charge, spin, etc.) and distributions (charge, current, momentum, one-dimensional and three-dimensional parton distribution functions, etc.). Lepton scattering, particularly electron scattering, has been proven to be a powerful microscope in probing the nucleon structure. In this paper, we will focus on the experimental status of the generalized parton distributions (GPDs) and the transverse momentum dependent parton distribution functions (TMDs), which provide three-dimensional imagings of the partonic structure of the nucleon, and uncover the rich dynamics of QCD.

\section{Three-dimensional parton distributions}

Following the original discovery of the "proton spin crisis" by the European Muon Collaboration in the late 1980s [四], the proton spin puzzle however remains unsolved. In recent analyses, the fraction of the proton spin carried by the quark spin is now confirmed to be about $30 \%$ [2]. Due to the Wigner rotation effect [B] which relates the spinors in different frames, the quark spin in the proton rest frame will decompose into a spin part and an orbital angular momentum part in the infinite momentum frame or the light-cone formalism where the parton language is well defined [四]. Therefore, the measurement of quark orbital angular momenta plays an important role in understanding the proton spin puzzle, although the gluon is also expected to contribute a significant fraction [ []]. GPDs and TMDs, as three-dimensional extensions of collinear parton distribution functions (PDFs), are expected to provide qualitative and quantitative information about the partonic orbital angular momentum contribution to the proton spin. However, the decomposition of the proton spin into quark and gluon degrees of freedom is nontrivial, and is still under debates [6].

A unified framework to describe the partonic structures of nucleons is the generalized transverse momentum dependent parton distributions (GTMDs) [ [ $]$, which contain the most general one-parton information inside a nucleon. They are related to the Wigner distributions [ [ ] ] via a transverse Fourier transformation. GPDs can be obtained by integrating GTMDs or Wigner distributions over the transverse momentum, and TMDs can be obtained by setting GTMDs at zero 
transferred momentum point or by integrating Wigner distributions over the transverse coordinate. Both of them will reduce to collinear PDFs when all the transverse variables are integrated out.

As all one-parton distribution information is encoded in GTMDs, one may obtain the quark orbital angular momentum from the GTMD via the relation,

$$
L_{q}=-\int d x d^{2} \boldsymbol{k}_{\perp} \frac{\boldsymbol{k}_{\perp}^{2}}{M^{2}} F_{1,4}^{q}\left(x, \boldsymbol{k}_{\perp}, \boldsymbol{\Delta}_{\perp}=0\right),
$$

where $x$ is the longitudinal momentum fraction, $\boldsymbol{k}_{\perp}$ is quark intrinsic transverse momentum, and $\boldsymbol{\Delta}_{\perp}$ is the transferred transverse momentum, which is the Fourier conjugate of quark intrinsic transverse coordinate $\boldsymbol{b}_{\perp}$. The GTMD $F_{1,4}$ represents the unpolarized quark distribution inside a longitudinally polarized nucleon. It depends on the choice of the gauge link that connects the field operators at different positions. A straight path gauge link relates $F_{1,4}$ to the kinetic orbital angular momentum, and a light-cone staple-like path gauge link relates it to the canonical orbital angular momentum [Q]. With a Fourier transformation, the relation can be expressed in terms of the Wigner distribution $\rho_{\mathrm{LU}}\left(x, \boldsymbol{k}_{\perp}, \boldsymbol{b}_{\perp}\right)$ [ய]].

GPDs as combined longitudinal momentum-transverse coordinate distribution functions are related to orbital angular momenta. One well-known relation is between the total quark angular momentum and the sum of two generalized (or gravitational) form factors, which are the second moments of the GPDs, $H^{q}$ and $E^{q}$. Together with quark helicity distributions that describe the quark spin part, one can obtain the orbital angular momentum via the GPDs as

$$
L_{q}=\int d x\left[x H^{q}(x, 0,0)+x E^{q}(x, 0,0)-\tilde{H}^{q}(x, 0,0)\right]
$$

A transverse counterpart of this relation was also proposed [ए]]. With QCD equation of motion, quark orbital angular momentum can be related to a twist-three GPD as [ए2]

$$
L_{q}=-\int d x x G_{2}^{q}(x, 0,0)
$$

The integration of GPDs over the longitudinal momentum gives the form factors, whose Fourier transformation provide transverse maps in the impact parameter space. In polarized situations, the distortion of the impact parameter distributions reflects the correlation with spins.

TMDs as complete momentum space distribution functions can also unveil the information of orbital angular momenta. The pretzelosity TMD $h_{1 T}^{\perp}\left(x, \boldsymbol{k}_{\perp}\right)$ is proposed as a quantity to access quark orbital angular momentum through

$$
L_{q}=-\int d x d^{2} \boldsymbol{k}_{\perp} \frac{\boldsymbol{k}_{\perp}^{2}}{2 M^{2}} h_{1 T}^{\perp q}\left(x, \boldsymbol{k}_{\perp}\right)
$$

which was first observed from spectator model calculations [[13] and then proven valid for all spherically symmetric situations [14]]. More generally, the pretzelosity distribution is the overlap between the wave functions differing by two units of orbital angular momentum. Thus nonvanishing pretzelosity distributions support nonzero values of quark orbital angular momenta. Similar situations apply to the worm-germ TMDs, $g_{1 T}^{q}$ and $h_{1 L}^{q}$, which are the overlap between the wave functions differing by one unit of orbital angular momentum. Besides, the correlation between 
TMDs and GPDs, such as the lensing relation between the Sivers function $f_{1 T}^{\perp q}$, a time-reversal odd TMD, and the GPD $E^{q}[[5]$, will also help access the information about orbital angular momenta.

Experimentally, it is currently not feasible to measure GTMDs or Wigner distributions, although some process was very recently proposed to access Wigner distributions in particular kinematic regions [ㅈ]. Fortunately, the theoretical framework has been established to access GPDs and TMDs, and certain processes have been utilized in measurements already.

A "golden channel" to measure GPDs is the deeply virtual Compton scattering (DVCS) process. It up to now provides the cleanest information about GPDs. In DVCS, GPDs contribute to the Compton form factors via weighted integrals, but only the cross-over line, where the longitudinal momentum fraction $x$ is equal to the skewness variable $\xi$, is directly measured. Another widely used process to access GPDs is the deeply virtual meson production (DVMP). The interpretation for DVMP is more complicated, since the GPD convolutes with the meson distribution amplitude, which is still not well known. Apart from these two processes, the double DVCS and the timelike Compton scattering (TCS) processes are complementary approaches to measure GPDs. Besides, form factors, which are the moments of GPDs, can also provide information or constraints on GPDs.

The semi-inclusive deep inelastic scattering (SIDIS) is widely used to measure TMDs. In this process, TMDs enter the structure functions via convolutions with fragmentation functions. With the universality, the fragmentation functions can be obtained from $e^{+} e^{-}$annihilations. The Drell-Yan process, as well as the $Z^{0} / W^{ \pm}$production process, is a complementary method to access TMDs. Its structure functions only contain the convolution of two TMDs. The comparison between SIDIS and Drell-Yan processes serves as a test of the universality of TMDs. The sign change prediction of the time-reversal odd TMDs, such as the Sivers function and the Boer-Mulders function, between SIDIS and Drell-Yan processes should also be examined experimentally.

A driving interest of the study of transversely polarized SIDIS processes is to measure quark transversity distributions, which are the transverse counterpart of helicity distributions. Due to the chiral odd property, the transversity distribution should be measured by coupling to another chiral odd quantity, such as the Collins fragmentation function or the dihadron fragmentation function. It can also be measured through the transversely polarized Drell-Yan process by convoluting with another chiral odd distribution function. The first moment of the transversity distribution is the tensor charge, which is a fundamental QCD quantity of the nucleon. It describes the transversely polarized quark number induced by the transverse polarization of the nucleon. In addition, the determination of the tensor charge, together with the upper limit on neutron electric dipole moment, will provide constraints on new physics beyond standard model [ㅁ]].

\section{Present status of GPD and TMD measurements}

During the last decade, many experimental efforts have been made to access three-dimensional tomographies of the nucleon. Both unpolarized and polarized experiments were carried out.

The unpolarized and the beam polarized $e p \rightarrow e p \gamma$ cross sections in the DVCS regime were first measured by JLab Hall A [ए8], and the improved analysis including more data was recently published [ए9]. The results showed clear deviation from the Bethe-Heitler process, and indicated a large contribution from the DVCS terms. The CLAS Collaboration performed the exclusive photon 
production from a longitudinal polarized proton target. Single beam, single target, and double spin asymmetries were measured in a wide kinematic range [20]. The cross section results were also obtained recently [2]]. A complete set of beam spin, beam charge, and target spin asymmetries were measured by HERMES [22].

The Compton form factors can be extracted from the data [23]. This is a step towards GPDs. As lacking of a multidimensional scan, the extraction of GPDs is usually based on some assumptions inspired by model calculations. The knowledge of collinear PDFs and form factors may also help constrain the functional form. There are already some GPD parameterizations that are in agreement with the existing world data [24].

As a complementary process, the exclusive meson production process has been measured in many experiments. Due to the relatively large cross section, the exclusive vector meson production was the most studied. Such measurements were carried out by the H1 [25], ZEUS [26], HERMES [27], CLAS [28], and other collaborations. The pseudoscalar meson production was also measured in some experiments [29, [3] ]. Many phenomenological models have been used to describe these data.

The exploration on TMDs was pioneered by HERMES with the first measurement of single spin asymmetries in SIDIS on a transversely polarized proton target [B]], and followed by COMPASS with the measurement on a transversely polarized deuteron target [32]. The azimuthal modulation due to the Collins effect was used to extract the transversity distribution. The other azimuthal modulations, such as the Sivers asymmetry and the pretzelosity asymmetry, were also measured. Although some results are still in preliminary forms, all the 16 azimuthal modulations in SIDIS on proton and deuteron targets have been measured. Physicists in JLab Hall A carried out the first SIDIS experiment on a transversely polarized neutron $\left({ }^{3} \mathrm{He}\right)$ target. The target polarization with a $12 \mu \mathrm{A}$ beam and a spin flip rate of every 20 minutes reached a new world record of 55\% 60\%. Single spin and double spin asymmetries were published [B3]. The neutron data improved the efficiency of flavor separations despite relatively large uncertainties. The result of the unpolarized cross section of SIDIS on a ${ }^{3} \mathrm{He}$ target was recently obtained [34]. It serves as the base for the understanding of the asymmetry data. Within the uncertainties, the azimuthal modulations are consistent with zero.

The $e^{+} e^{-}$annihilation experiments by Belle [35], BaBar [36], and BESIII [B37] provide a complementary measurement to help disentangle the distribution and the fragmentation functions in SIDIS observables.

In the meanwhile, Drell-Yan process was carried out by Fermilab [B8] and RHIC. The transverse single spin asymmetry was recently measured in $Z^{0} / W^{ \pm}$production from $p p_{\uparrow}$ collisions by STAR [39]. This provides a first attempt to test the sign change prediction of the Sivers function.

With the data collected during last decade, TMDs have been parameterized by many groups. In the stage of exploration, most parameterizations were obtained without the TMD evolution, and much attention was paid to the polarized TMDs. Thanks to the development of the theoretical formalism in recent years, we are currently at the "phase transformation point" to precision measurement. The TMD evolution effect has been taken into account in some recent parameterizations of the unpolarized TMD, the Sivers function, and the transversity distribution [40, 4]]. Since unpolarized TMDs are the base for all extractions of polarized TMDs, more careful studies are required.

Besides, the transversity distribution was also extracted from the dihadron data in the frame- 
work of collinear factorization [42]. It is a complementary approach to the extraction from the SIDIS data in the framework of the TMD factorization.

\section{Future experiments}

In the coming decade, we expect to have more precise measurements of GPDs and TMDs to have better knowledge of the three-dimensional structures of the nucleon and the QCD dynamics.

The JLab $12 \mathrm{GeV}$ upgrade provides an ideal opportunity to access nucleon three-dimensional tomographies in the valence quark region [43]]. The DVCS experiments in Hall A (E12-06-114), Hall B (E12-06-119), and Hall C (E12-13-010) on unpolarized proton targets with different beam energies will cover a wide kinematics range. The DVCS experiment in Hall B on a neutron target (E12-11-003) will help flavor separations. The DVCS experiments in Hall B on longitudinally (E12-06-119) and transversely (E12-12-010) polarized proton targets will measure target single spin and beam-target double spin asymmetries. Thus the DVCS will be measured with a complete set of polarization configurations. The run-group TCS experiment in Hall A with SoLID will provide a complementary measurement of GPDs.

The SIDIS experiments on a transversely polarized ${ }^{3} \mathrm{He}$ target in Hall A with SBS (E12-09018) is an upgrade of the $6 \mathrm{GeV}$ SIDIS experiment (E06-010) with a wider kinematic coverage and a higher luminosity. The statistics will be enough to have three-dimensional bins. The SoLID SIDIS program will combine a high luminosity and a large acceptance to have an unprecedented precision measurement of TMDs in the valence quark region. The three approved SIDIS experiments are respectively with a transversely polarized neutron $\left({ }^{3} \mathrm{He}\right)$ target (E12-10-006), a longitudinally polarized neutron $\left({ }^{3} \mathrm{He}\right)$ target (E12-11-107), and a transversely polarized proton target (E12-11-108). The high statistics will allow for four-dimensional bins. The scan on each variable with the other three variable fixed will become possible in a certain region. Together with the approved SIDIS experiment in Hall B on longitudinally polarized proton and deuteron targets with CLAS12 (E12-09-009), we will have a complete SIDIS measurement with all polarization configurations on both proton and neutron targets. The impact of SoLID SIDIS program was recently studied with the extraction of transversity distributions as an example, and it is expected to improve the uncertainties by one order of magnitude [44]. The Hall C SIDIS program is aiming to test the factorization framework by scanning in different variables (E12-13-007, E12-09-017, E12-09-002, E12-13-010, E12-06-104). Neutral pion will also be measured in some experiments.

In addition, future Drell-Yan data from COMPASS will help disentangle the fragmentation functions in SIDIS measurements, and also provide valuable information on pion TMDs.

\section{Summary}

The proton spin puzzle remains unsolved after more than 25 years. Three-dimensional imagings encoded in GPDs and TMDs are expected to help solve this puzzle and uncover the rich dynamics of QCD. During the last decade, many experiments were carried out to access GPDs and TMDs, and significant progresses were made. However, we currently still have very rough knowledge about them. Although there are already parameterizations of certain distributions, the 
precision is far from a satisfactory level. The experiments planned in the coming decade, especially those at the $12 \mathrm{GeV}$ upgraded JLab, will provide a unique stage to unveil three-dimensional structures of the nucleon in the valence quark region.

\section{Acknowledgment}

This work is supported in part by U.S. Department of Energy under contract No. DEFG0203ER41231. It is also supported in part by the Duke Kunshan University.

\section{References}

[1] J. Ashman et al. (European Muon Collaboration), Phys. Lett. B 206, 364(1988); J. Ashman et al. (European Muon Collaboration), Nucl. Phys. B 328, 1 (1989),

[2] N. Sato et al. (Jefferson Lab Angular Momentum Collaboration), Phys. Rev. D 93, 074005 (2016).

[3] E.P. Wigner, Annals Math. 40, 149 (1939) [Nucl. Phys. Proc. Suppl. 6, 9 (1989)].

[4] B.-Q. Ma, J.Phys. G 17, L53 (1991); B.-Q. Ma and Q.-R. Zhang, Z. Phys. C 58, 479 (1993).

[5] D. de Florian, R. Sassot, M. Stratmann, and W. Vogelsang, Phys. Rev. Lett. 113, 012001 (2014).

[6] E. Leader and C. Lorcé, Phys. Rep. 541, 163 (2014); M. Wakamatsu, [nt. J. Mod. Phys. A 29, $1430012(2014)$.

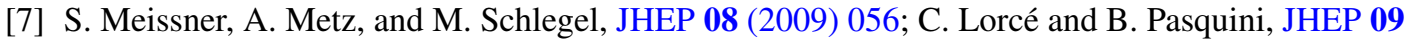
$2013) 138$.

[8] C. Lorcé and B. Pasquini, Phys. Rev. D 84, 014015 (2011),

[9] C. Lorcé, Phys. Rev. D 87, 034031 (2013); C. Lorcé, Phys. Lett. B 719, 185 (2013),

[10] C. Lorcé, B. Pasquini, X. Xiong, and F. Yuan, Phys. Rev. D 85, 114006 (2012).

[11] X. Ji, X. Xiong, and F. Yuan, Phys. Rev. Lett. 109, 152005 (2012); E. Leader and C. Lorcé, Phys. Rev. Lett. 111,039101(2013).

[12] D.V. Kiptily and M.V. Polyakov, Eur. Phys. J. C 37, 105 (2004).

[13] J. She, J. Zhu, and B.-Q. Ma, Phys. Rev. D 79, 054008 (2009).

[14] C. Lorcé and B. Pasquini, Phys. Lett. B 710, 486 (2012).

[15] A. Bacchetta et al., JHEP02 (2007)093.

[16] Y. Hatta, B.-W. Xiao, and F. Yuan, Phys. Rev. Lett. 116, 202301 (2016).

[17] H. Gao et al., The Universe 3, no. 2, 18 (2015).

[18] C.M. Camacho et al. (Jefferson Lab Hall A and Hall A DVCS Collaborations), Phys. Rev. Lett. 97, $262002(2006)$.

[19] M. Defurne et al. (Jefferson Lab Hall A Collaboration), Phys. Rev. C 92, 055202 (2015).

[20] F.X. Girod et al. (CLAS Collaboration), Phys. Rev. Lett. 100, 162002 (2008); S. Pisano et al. (CLAS Collaboration), Phys. Rev. D91, 052014(2015).

[21] H.S. Jo et al. (CLAS Collaboration), Phys. Rev. Lett. 115, 21, 212003 (2015), 
[22] A. Airapetian et al. (HERMES Collaboration), Phys. Rev. Lett. 87, 182001 (2001); A. Airapetian et al. (HERMES Collaboration), Phys. Rev. D 75, 011103 (2007); A. Airapetian et al. (HERMES Collaboration), एHEP 06 (2008) 066; A. Airapetian et al. (HERMES Collaboration), एHEP II (2009) 083; A. Airapetian et al. (HERMES Collaboration), Phys. Rev. C 81, 035202 (2010); A. Airapetian et al. (HERMES Collaboration), JHEP 06 (2010) 019; A. Airapetian et al. (HERMES Collaboration), Phys. Lett. B 704, 15 (201); A. Airapetian et al. (HERMES Collaboration), WHEP07(2012)032.

[23] K. Kumerički, D. Müller, and M. Murray, Phys. Part. Nucl. 45, 723 (2014); H. Moutarde, Phys. Rev. D79,094021(2009).

[24] G.R. Goldstein, J.O. Hernandez, and S. Liuti, Phys. Rev. D 84, 034007 (2011); G.R. Goldstein, J.O. Hernandez, and S. Liuti, Phys. Rev. D 91, 114013(2015).

[25] C. Adloff et al. (H1 Collaboration), Eur. Phys. J. C 13,371 (2000); F. D. Aaron et al. (H1 Collaboration), एHEP 05 (2010)032.

[26] S. Chekanov et al. (ZEUS Collaboration), Nucl. Phys. B 718, 3 (2005).

[27] A. Airapetian et al. (HERMES Collaboration), Eur. Phys. J. C 17,389(2000).

[28] C. Hadjidakis et al. (CLAS Collaboration), Phys. Lett. B 605, 256 (2005); S.A. Morrow et al. (CLAS Collaboration), Eur. Phys. J. A 39, 5 (2009); L. Morand et al. (CLAS Collaboration), Eur. Phys. J. A 24,445(2005).

[29] A. Airapetian et al. (HERMES Collaboration), Phys. Lett. B 659, 486 (2008).

[30] I. Bedlinskiy et al. (CLAS Collaboration), Phys. Rev. Lett. 109, 112001 (2012).

[31] A. Airapetian et al. (HERMES Collaboration), Phys. Rev. Lett. 94, 012002 (2005).

[32] V.Y. Alexakhin et al. (COMPASS Collaboration), Phys. Rev. Lett. 94, 202002 (2005).

[33] X. Qian et al. (Jefferson Lab Hall A Collaboration), Phys. Rev. Lett. 107, 072003 (2011); Y. Zhang et al. (Jefferson Lab Hall A Collaboration), Phys. Rev. C 90, 055209 (2014); Y.X. Zhao et al. (Jefferson Lab Hall A Collaboration), Phys. Rev. C 90, 055201 (2014); J. Huang et al. (Jefferson Lab Hall A Collaboration), Phys. Rev. Lett. 108, 052001(2012).

[34] X. Yan et al. (Jefferson Lab Hall A Collaboration), arXiv: 1610.02350 nnucl-ex].

[35] R. Seidl et al. (Belle Collaboration), Phys. Rev. D 78, 032011 (2008) [Erratum: Phys. Rev. D 86, 039905 (2012)]; A. Vossen et al. (Belle Collaboration), Phys. Rev. Lett. 107, 072004 (2011).

[36] J.P. Lees et al. (BaBar Collaboration), Phys. Rev. D 90, 052003 (2014).

[37] M. Ablikim et al. (BESIII Collaboration), Phys. Rev. Lett. 116, 042001 (2016).

[38] L. Y. Zhu et al. (NuSea Collaboration), Phys. Rev. Lett. 99, 082301 (2007).

[39] L. Adamczyk et al. (STAR Collaboration), Phys. Rev. Lett. 116, 132301 (2016).

[40] M.G. Echevarria, A. Idilbi, Z.-B. Kang, and I. Vitev, Phys. Rev. D 89, 074013 (2014).

[41] Z.-B. Kang, A. Prokudin, P. Sun, and F. Yuan, Phys. Rev. D 93, 014009 (2016).

[42] M. Radici, A. Courtoy, A. Bacchetta, and M. Guagnelli, WHEP05 (2015) 123.

[43] H. Gao et al., Eur. Phys. J. Plus 126, 2 (2011).

[44] Z. Ye et al., arXiv: 1609.02449 [hep-ph]. 